\title{
BACKWARD ERRORS AND PSEUDOSPECTRA FOR STRUCTURED NONLINEAR EIGENVALUE PROBLEMS
}

\author{
SK. SAFIQUe Ahmad And VolKer MeHrmann
}

Abstract. Minimal norm structured perturbations (backward errors) are constructed such that an approximate eigenpair of a nonlinear eigenvalue problem is an exact eigenpair of an appropriately perturbed problem. Structured and unstructured backward errors are compared. These results extend previous results for (structured) matrix polynomials to more general functions.

Mathematics subject classification (2010): 65F15, 15A18, 65F35, 15A12.

Keywords and phrases: nonlinear eigenvalue problem, backward error, symmetric/skew symmetric eigenvalue problem, Hermitian/skew-Hermitian eigenvalue problem.

\section{REFERENCES}

[1] B. ADHIKARI, Backward perturbation and sensitivity analysis of structured polynomial eigenvalue problem, PhD thesis, IIT Guwahati, Dept. of Mathematics, 2008.

[2] B. Adhikari And R. Alam, Structured backward errors and pseudospectra of structured matrix pencils, SIAM J. Matrix Anal. Appl., 31: 331-359, 2009.

[3] B. ADHIKARI AND R. AlAM, On backward errors of structured polynomial eigenproblems solved by structure preserving linearizations, Linear Algebra Appl., 434: 1989-2017, 2011.

[4] S. S. AHMAD, Pseudospectra of matrix pencils and their applications in the perturbation analysis of eigenvalues and eigendecompositions, PhD thesis, IIT Guwahati, Dept. of Mathematics, 2007.

[5] S. S. Ahmad And R. Alam, Pseudospectra, critical points and multiple eigenvalues of matrix polynomials, Linear Algebra Appl., 430: 1171-1195, 2009.

[6] S. S. AHMAD, R. ALAM, AND R. B YERS, On pseudospectra, critical points and multiple eigenvalues of matrix pencils, SIAM J. Matrix Anal. Appl., 31: 1915-1933, 2010.

[7] S. S. Ahmad and V. Mehrmann, Perturbation analysis for complex symmetric, skew symmetric, even and odd matrix polynomials, Electr. Trans. Num. Anal., 38: 275-302, 2011.

[8] S. S. Ahmad AND V. Mehrmann, Backward errors for eigenvalues and eigenvectors of Hermitian, skew-Hermitian, H-even and H-odd matrix polynomials, Lin. Multilin. Alg., 1: 1-23, 2012.

[9] T. Betcke, N. J. Higham, V. Mehrmann, C. Schröder, and F. Tisseur, NLEVP: A Collection of Nonlinear Eigenvalue Problems, J. ACM TOMS, 39 (2): 7:1-7:28, February 2013.

[10] S. BORA, Structured eigenvalue condition number and backward error of a class of polynomial eigenvalue problems, SIAM J. Matrix Anal. Appl., 31: 900-917, 2009.

[11] S. Bora AND V. MeHRMann, Perturbation theory for structured matrix pencils arising in control theory, SIAM J. Matrix Anal. Appl., 28: 148-169, 2006.

[12] S.-H. Chou, T.-M. HuAng, W.-Q. HuAnG, AND W.-W. Lin, Efficient Arnoldi-type algorithms for rational eigenvalue problems arising in fluid-solid systems, J. Comput. Phys., 230: 2189-2206, 2011.

[13] C. DAVIS, W. KAHAN, AND H. WeInBERGER, Norm-preserving dialations and their applications to optimal error bounds, SIAM J. Math. Anal., 19: 445-469, 1982.

[14] N. H. Du, V. H. Linh, V. Mehrmann, And D. D. Thuan, Stability and robust stability of linear time-invariant delay differential-algebraic equations, SIAM J. Matrix Anal. Appl., 34: 1631-1654, 2013.

[15] C. Effenberger, D. Kressner, And C. Engström, Linearization techniques for band structure calculations in absorbing photonic crystals, Int. J. Numer. Meth. Engineering, 89: 180-191, 2012. 
[16] I. Gohberg, P. LANCASTER, AND L. Rodman, Spectral analysis of self adjoint matrix polynomials, Ann. of Math. (2), 112: 33-71, 1980.

[17] G. H. Golub and C. F. Van Loan, Matrix Computations, The Johns Hopkins University Press, Baltimore, third edition, 1996.

[18] K. Green And T. Wagenknecht, Pseudospectra and delay differential equations, J. Comput. Appl. Math., 196: 567-578, 2006.

[19] D. J. Higham AND N. J. Higham, Structured backward error and condition of generalized eigenvalue problems, SIAM J. Matrix Anal. Appl., 20: 493-512, 1998.

[20] N. J. Higham, The Accuracy and Stability of Numerical Algorithims, SIAM, Philadelphia, USA, 1996.

[21] N. J. Higham, R. C. LI, AND F. TiSSEUR, Backward error of polynomial eigenproblems solved by lineariation, SIAM J. Matrix Anal. Appl., 29: 1218-1241, 2007.

[22] N. J. Higham, D. S. Mackey, AND F. Tisseur, The Conditioning of Linearizations of Matrix Polynomials, SIAM J. Matrix Anal. Appl., 28: 1005-1028, 2006.

[23] N. J. Higham AND F. TISSEUR, More on pseudospectra for polynomial eigenvalue problems and applications in control theory, Linear Algebra Appl., 351-352: 435-453, 2002.

[24] T. M. HWANG, W. W. Lin, J. L. LiU, AND W. WANG, Jacobi-Davidson methods for cubic eigenvalue problems, Numer. Lin. Alg. Appl., 12: 605-624, 2005.

[25] T. M. HWANG, W. W. Lin, W. C. WANG, AND W. WANG, Numerical simulation of three dimensional quantom dot, J. Comp. Physics, 196: 208-232, 2004.

[26] E. Jarlebring, The Spectrum of Delay-Differential Equations: Numerical Methods, Stability and Perturbation, Dissertation thesis, TU Braunschweig, 2008.

[27] M. KAROW, $\mu$-values and spectral value sets for linear perturbation classes defined by a scalar product, SIAM J. Matrix Anal. Appl., 32: 845-865, 2011.

[28] M. Karow, E. Kokiopoulou, And D. Kressner, On the computation of structured singular values and pseudospectra, Systems Control Lett., 59: 122-129, 2010.

[29] L. MAZURENKO AND H. Voss, Low rank rational perturbations of linear symmetric eigenproblems, Z. Angew. Math. Mech., 86: 606-616, 2006.

[30] R.-C. LI, W.-W. Lin, AND C.-S. WANG, Structured backward error for palindromic polynomial eigenvalue problems, Numer. Math., 116: 95-122, 2010.

[31] D. S. Mackey, N. Mackey, C. Mehl, and V. Mehrmann, Structured polynomial eigenvalue problems: Good vibrations from good linearizations, SIAM J. Matrix Anal. Appl., 28: 1029-1051, 2006.

[32] D. S. Mackey, N. Mackey, C. Mehl, and V. Mehrmann, Vector Spaces of Linearizations for Matrix Polynomials, SIAM J. Matrix Anal. Appl., 28: 971-1004, 2006.

[33] V. Mehrmann AND C. SCHRÖDER, Nonlinear eigenvalue and frequency response problems in industrial practice, J. Math. in Industry, 1:7, 2011.

[34] V. Mehrmann AND H. Voss, Nonlinear eigenvalue problems: a challenge for modern eigenvalue methods, GAMM Mitteilungen, 27: 121-152, 2004.

[35] W. Michiels, K. Green, T. Wagenknecht, and S. Niculescu, Pseudospectra and stability radii for analytic matrix functions with application to time-delay systems, Linear Algebra Appl., 418: 315-335, 2006.

[36] S. I. SOLOV'Ev, Preconditioned iterative methods for a class of nonlinear eigenvalue problems, Linear Algebra Appl., 415: 210-229, 2006.

[37] Y. SU AND Z. BAI, Solving rational eigenvalue problems via linearization, SIAM J. Matrix Anal. Appl., 32: 201-216, 2011.

[38] F. TISSEUR, Backward error and condition of polynomial eigenvalue problem, Linear Algebra Appl., 309: 339-361, 2000.

[39] F. Tisseur And N. J. Higham, Structured pseudospectra for polynomial eigenvalue problems, with applications, SIAM J. Matrix Anal. Appl., 23: 187-208, 2001.

[40] F. Tisseur AND K. MEerbergen, A survey of the quadratic eigenvalue problem, SIAM Rev., 43: 234-286, 2001.

[41] L. N. Trefethen And M. Embree, Spectra and Pseudospectra: The Behavior of Nonnormal Matrices and Operators, Princeton Univ. Press, Princeton, N.J., 2005.

[42] H. Voss, A rational spectral in fluid solid vibration, Electr. Trans. Num. Anal., 16: 94-106, 2003. 
[43] C. YAng, Solving Large-scale Eigenvalue problems in SciDAC Applications, J. Conference Series, 16: 425-434, 2005. 